\title{
Clipboard
}

\section{Bone marrow stromal cells and multi-lineage differentiation}

Bone marrow consists of progenitor cells that give rise to stromal cells and/or haematopoietic cells. Although there have been indirect evidences that progenitor cells in the bone marrow could be common to stromal and hematopoietic cells, there has been no direct evidence for this conclusion. Stromal cells were thought to be multipotential by several in vitro experiments however there has been a debate weather multiple stromal cells exist for different lineages. Hence, a major question has been whether there are multiple stromal cell types for each differentiation pathway or whether one stromal cell type is capable of generating many phenotypes. This has been recently addressed in an elegant study using a single-cell-microarray approach (Seshi et al 2003). The gene expression profile of single stromal cells isolated from the human bone marrow after culture expansion followed by laser-capture was compared to that of pooled stromal cells. Microarray data are faught with problems of interpretation (see below). The authors have managed to overcome these difficulties and have arrived at a simply stated but important conclusion.

Seshi et al (2003) find that a single stromal cell can express transcripts characteristic of muscle cells, bone cells, fat cells, epithelial cells, endothelial cells and neural or glial cells. This suggests that a single non-haematopoietic stromal cell is capable of differentiating into multiple lineages: it is not necessary to have multiple stromal cells for multiple lineages. In addition, isolated single stromal cells also express markers typically associated with normal and neoplastic haematopoietic cells. The hypothesis that a single stromal cell is multipotential has also been put forward by Rosenberger (2003) while interpreting the data from ES cell differentiation, which suggests that uncommitted ES cells co-express genes for different lineages. The interpretation of multiple lineage pathways of a single cell could also be relevant for cancer biology, because stem cells may be seeds for cancer development (Marx 2003).

Besides the thought-provoking result, the paper of Seshi et al demonstrates a novel technique for generating single cell microarray data and makes use of several statistical methods to filter and refine the output. In analysing the findings generated by microarray experiments, a careful application of normalization techniques and statistical methods is critical if one wishes to be confident of arriving at a reasonably accurate picture of gene regulation. The present study is based on an amplification method for generating cRNA probes using T7 RNA polymerase for microarray hybridizations. It is well known that there is a tendency for individual transcripts to be amplified differentially. Consequently the data can generally be subjected to a qualitative interpretation alone. Seshi et al pay heed to this problem and indicate means for the standardization of the data.

In conclusion, Seshi et al (2003) have successfully demonstrated the insights that can be derived from mapping the transcriptome of a single cell and provide evidence that bone marrow stromal cells are relatively homogeneous but multipotent - and not necessarily heterogeneous in a functional sense.

\section{References}

Marx J 2003 Mutant stem cells may seed cancer; Science 301 1308-1310

Rosenberger R F 2003 The choice of cell lineages during the in vitro differentiation of mammalan embryonic stem cells; J. Theor. Biol. 223 387-389

Seshi B, Kumar S and King D 2003 Multilineage gene expression in human bone marrow stromal cells as evidenced by single-cell micro array analysis; Blood Cells Molecules Diseases 31 268-285

PATURU KONDAIAH Molecular Reproduction, Development and Genetics Department, Indian Institute of Science, Bangalore 560 012, India

(Email,paturu@mrdg.iisc.ernet.in) 